\title{
Non-linearity of apparent mass for multi-element bodies
}

\author{
Kenneth O. Granlund* and Michael V. OL ${ }^{\dagger}$ \\ U.S. Air Force Research Laboratory, Wright-Patterson Air Force Base, OH, 45433 \\ Luis P. Bernal ${ }^{\ddagger}$ \\ University of Michigan, Ann Arbor, MI, 48109
}

\section{Nomenclature}

$b \quad$ plate $\operatorname{span}(450 \mathrm{~mm})$

c plate chord $(75 \mathrm{~mm})$

d plate spacing

$h \quad$ oscillation amplitude

$h_{0} \quad$ normalized oscillation amplitude $(=h / c)$

$\rho \quad$ fluid density $\left(1000 \mathrm{~kg} / \mathrm{m}^{3}\right)$

\section{Introduction}

Classical, ${ }^{1-5}$ and recent ${ }^{6-11}$ expositions of aerodynamic force history of unsteady incompressible problems segregate the forces into those due to the relative acceleration between the body immersed in a fluid, and everything else. Loads due to acceleration are referred to as inertial, noncirculatory, or apparent (or added) mass, since their cause is imputed to acceleration of a volume of bulk-fluid of some density (hence, inertia), to causes other than circulation, or to what mathematically looks like a slug of fluid moving with the accelerating body, respectively. The everything else lumps together forces due to bound or shed circulation, forces due to flow separation, and viscous traction. Ultimately all aerodynamic loads are from integration of pressure distribution and viscous traction, so the distinction into

*Aerospace Systems Directorate, AIAA Senior Member, kenneth.granlund@wpafb.af.mil

${ }^{\dagger}$ Aerospace Systems Directorate, AIAA Associate Fellow

${ }^{\ddagger}$ Department of Aerospace Engineering, AIAA Senior Member 
circulatory and noncirculatory loads is somewhat artificial. However, it is a useful artifice because the noncirculatory loads are asserted to be independent of geometric details such as sharp trailing edges supporting a Kutta condition, or dynamic conditions such as Reynolds number. Further, one seeks to understand how unsteady a given unsteady problem really is, and this can be beheld from the ratio of circulatory to noncirculatory forces. If circulatory and noncirculatory force histories can be superimposed, this supports an argument for linear superposition and hence for linearity. This would be particularly curious if the noncirculatory forces are the same in attached and in separated flows, for example for a flat plate with strong vortex shedding from its edges, and one without.

The simplest classical unsteady incompressible problem is that of a thin rigid flat plate oscillating harmonically in a direction normal to its chord. By taking the unsteady Bernoulli equation without gravity and body forces

$$
p=-\rho\left(\frac{\partial \phi}{\partial t}+\frac{u_{\theta}^{2}}{2}\right)
$$

and integrating over a circle with diameter c, we get the pressure force.

$$
F_{p}^{\prime}=-\rho \int_{0}^{2 \pi}\left(\frac{\partial \phi}{\partial t}+\frac{u_{\theta}^{2}}{2}\right) d \theta=\left\lceil\frac{\partial \phi}{\partial t}=\frac{\partial u}{\partial t} \cos \theta c\right\rceil=-\rho \pi \frac{c^{2}}{4} \frac{\partial u}{\partial t}
$$

If the cylinder is conformally mapped to a flat plate, the result in Equation 2 does not change. Thus, the constant of force proportional to acceleration of a flat plate body is equivalent to a circle of fluid encompassing the chord. Of course, the plates motion produces an unsteady pressure field propagating everywhere in the fluid, with intensity decreasing in going away from the plate. That the force happens to be equal to that of accelerating cylindrical mass of fluid around the plate is a convenient heuristic, and not an essential description of nature. But it has emerged as an appealing colloquial term, and we follow such treatment here.

In unsteady fluid mechanics force models, the fluid acceleratory term is derived from integrating the velocity potential in all of the fluid surrounding the body, assuming potential flow. High-rate, large excursion motions produce massive separation and palpably violate the potential flow assumption. For cases such as pitching and plunging airfoils, one finds unsteady potential-flow models to be remarkably successful regardless of motion frequency and up to motion amplitudes of appreciable fraction of airfoil chord. Granlund et al. ${ }^{11}$ observed independence of circulatory force from variation in the non-circulatory for a plate pitching from 0 to 90 degrees at the onset of the motion. However, the force after motion cessation at 90 degrees is different. We examine to what extent similar success of theoretical models with assumptions belied by observed reality nevertheless yield predictions fitting the observed data. 
One asks immediately regarding linear superposition: in the simplest such case, suppose that we have two plates adjacent to each other, some distance apart. Can the above solution for one plate, be superimposed to that of the second, for a combined solution? The immediate answer is no. Consider the schematic in Figure 1 where two plates are positioned far apart on the same chord line. Acceleration of one plate normal to the chord induces an acceleration of the surrounding fluid. Since the plates are far apart, the fluid acceleration of one has minimal effect on the other, and vice versa and thus the total force by both plates are the sum of the individual added mass multiplied by the acceleration.

If both plates are joined together producing a 2c chord plate, the pressure field from an acceleration of the plate now produces an equivalent added mass of twice that of the individual plates together, since the constant in Equation 2 is dependent on the chord squared. For the intermediate case of where the two plates are separated, but in close proximity to each other, the acceleration of fluid from one plate should now affect the other and the resulting equivalent added mass be somewhere in between the two aforementioned extrema. The question is how the combined added mass as a function of chord separation looks like, and whether it is affected by fluid viscosity, as the potential flow derivation is inviscid.

In the present work, we pursue experimental assessment of the noncirculatory solution for the problem in Figure 1, by varying the gap between the two plates from zero (a single plate of chord 2c) to 1 (two places of chord c, with gap of 1c). We also consider the case of nonzero free stream parallel to the plates. Questions include:

1. How well does the single-plate theoretical solution comport with measurement, for the baseline case?

2. How does the above degrade as motion amplitude is no longer small, violating the assumptions of the theory and evidently introducing appreciable circulatory effects (vortex shedding from the plate edges)?

3. Does nonzero free-stream leave the theoretical solution unaffected, again for very small and not so small amplitudes (in the latter there is presumably a bound circulation due to effective angle of attack of a plunging plate in nonzero free stream)?

4. How does the loading for two plates adjacent to each other vary with the gap between them?

5. Is the noncirculatory force indeed quadratic with the motion frequency? 

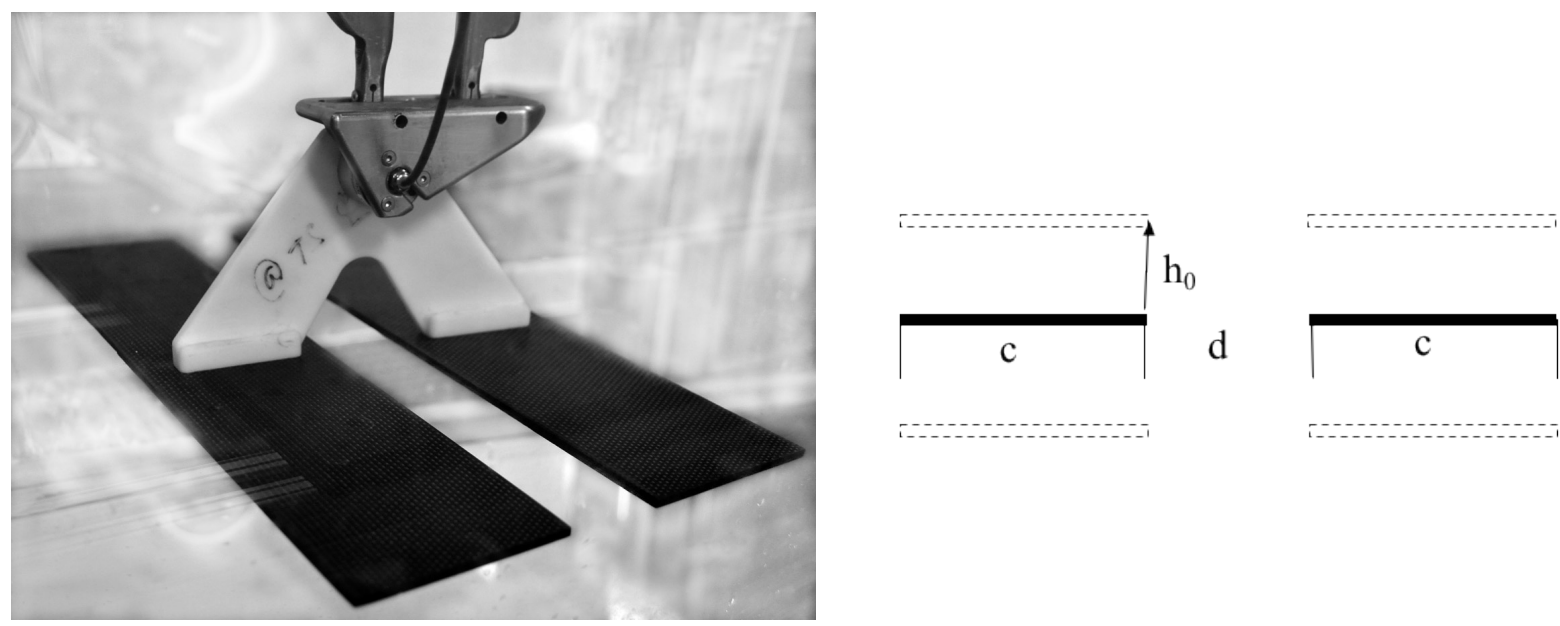

Figure 1: Two wall-to-wall carbon fiber flat plates mounted horizontally in the test section (left) and schematic of the setup with definition of variables (right).

\section{Experimental Setup}

Experiments are conducted at the U.S Air Force Research Lab, Aerospace Systems Directorate Horizontal Free-Surface Water Tunnel. The tunnel is fitted with a three-degree of freedom electric motion rig, controlled via a Galil DMC-4040 motion controller from preprogrammed scripts achieving $<0.1 \mathrm{~mm}$ linear- and $<0.2^{\circ}$ angular position error. Two vertical linear motors move the test article linearly normal to the test section free-stream direction (that is, in plunge). A third linear motor, mounted horizontally above the test section, produces streamwise displacement. The three in concert allow for a third degree of freedom, rotation or pitch about an axis parallel to the test articles span, with pivot point arbitrarily selectable.

Two carbon-fiber flat plates with $75 \mathrm{~mm}$ chord, $3 \mathrm{~mm}$ thickness and $0.45 \mathrm{~m}$ span are positioned coplanar, with a coupler piece connecting to the load cell shown in Figure 1. The gap between the plate tips and the test section sidewalls is $\approx 1 \mathrm{~mm}$. Chordwise spacing $d$ between the plates is taken as $0.1 \mathrm{c}, 0.2 \mathrm{c}, 0.5 \mathrm{c}$ and $1 \mathrm{c}$, by mounting the respective coupler piece. A case of single plate of chord $2 c$ serves as baseline. The sinusoidal amplitude of the oscillation of the plates is varied $0.01<h_{0}<0.05$. The higher range of amplitude is expected to produce appreciable vortex shedding, and one of our aims is to ascertain how this shedding mediates the theoretical potential-flow apparent-mass solution. In addition to twin-plate cases in quiescent fluid, a single $75 \mathrm{~mm}$ chord plate is also tested at $45^{\circ}$ angle of attack in freestream and oscillated normal to the plate in order to investigate the linearity and superpositionability of the apparent mass in bluff-body shedding.

According to Brennen, ${ }^{14}$ proximity of a free surface to an accelerating body, lesser than 4 times the body dimension will cause an increase in apparent mass with acceleration of the 
fluid. A distance between a free surface and a body of less than the body dimension will cause a decrease in apparent mass. Since the plates are oscillated at the centerline of the test section with exactly 4 chords to either the test section bottom wall and the free surface, it is expected that any boundary effects are minimized.
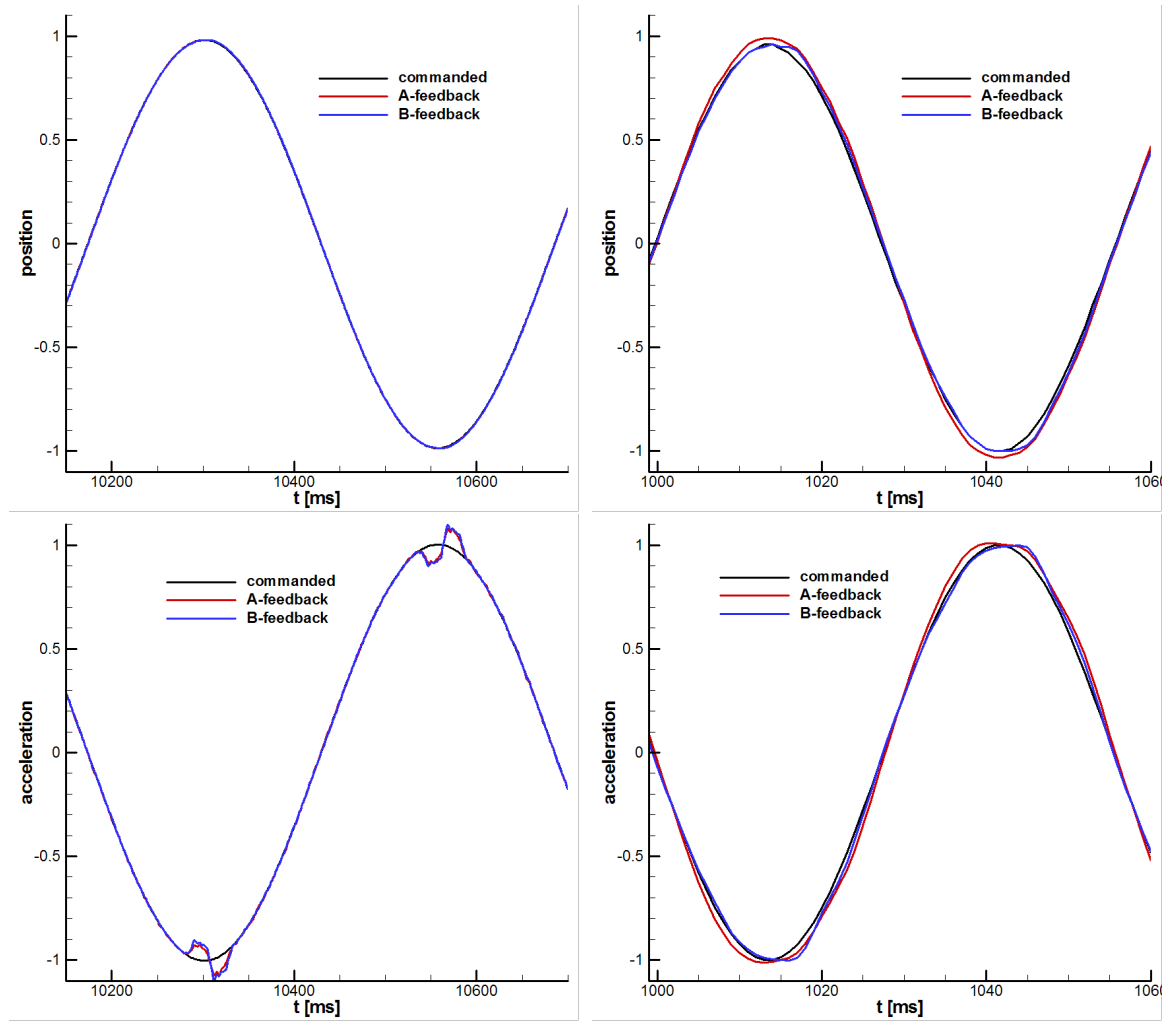

Figure 2: Typical motion kinematics, measured at the linear motor encoder-tape: $1 \mathrm{~Hz} 0.05 \mathrm{c}$ amplitude motion (left), and the complementary case, $9 \mathrm{~Hz} 0.01 \mathrm{c}$ amplitude motion (right); position (top row) and acceleration (bottom row). A- and B- are front and rear vertical motor plunge rods visible in top of Figure 1.

We first characterize the imposed kinematics, since the theoretical model assumes a perfect sinusoid. Figure 1 show the linear vertical electric motor positions (A is front and B is rear in the top of Figure 1) and acceleration as the twice differentiated position signal with an 11 point moving average filter before each differentiation. Position and acceleration are normalized to 1 . Both sets of curves curves show that position and acceleration are followed accurately and to the same magnitude as the prescribed motion. The low frequency motion has slight jitter at the acceleration peaks which is attenuated below observable as the frequency is increased. Overall though, there is no evidence of deviation from prescribed motion that can cause unexpected results from acceleration of the flat plates in fluid.

Force measurements were with an ATI Nano25 IP68 6-component balance, sampled at $1 \mathrm{kHz}$ and low- pass filtered at $34 \mathrm{~Hz}$ in hardware, and then at 50x of the motion frequency using a 4th order Chebychev II low-pass filter in Matlab. The data is synchronized exter- 

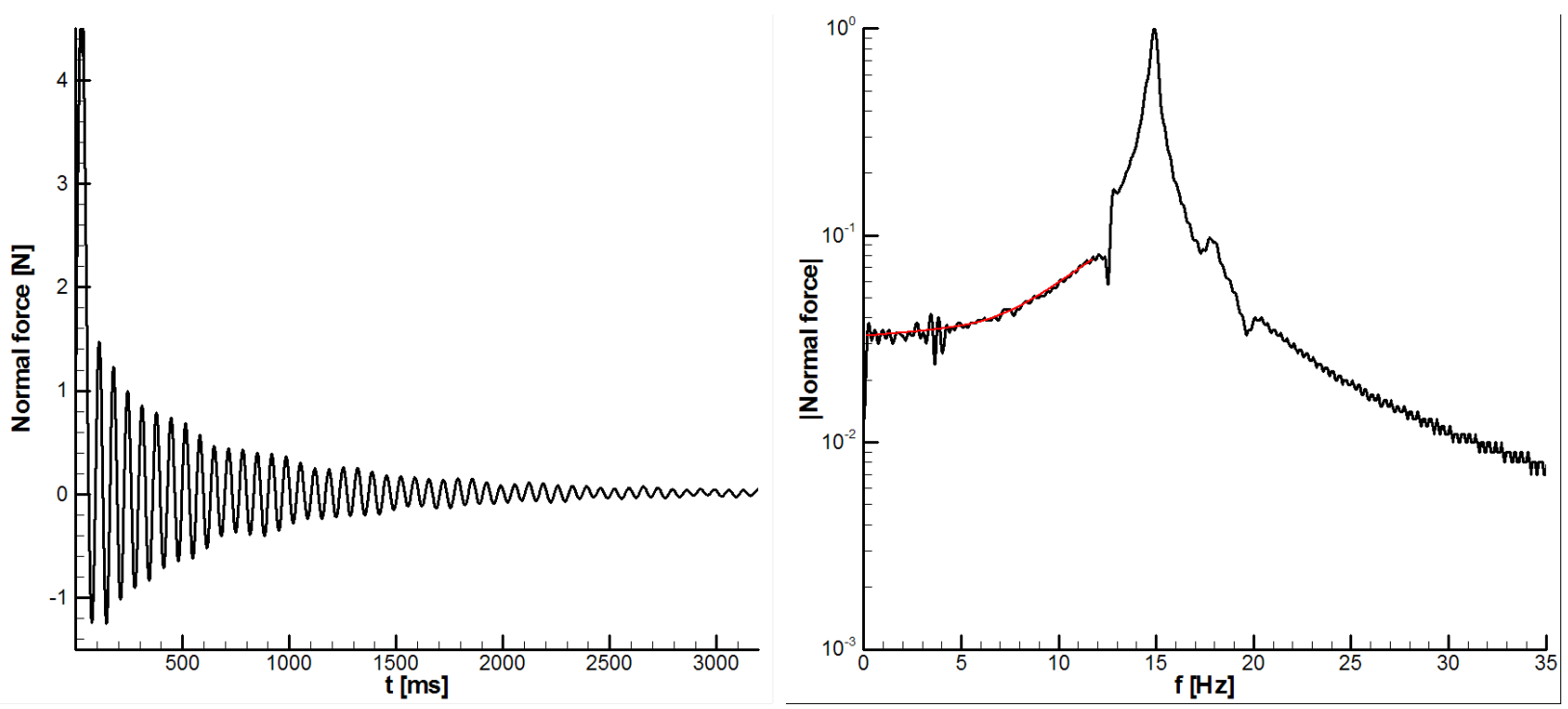

Figure 3: Results of mechanical strike-test of the plate coupler piece, recorded at the load cell, with the linear motors energized but not moving; temporal-history (left) and FFT (right).

nally via a trigger pulse from the DMC-4040 motion controller at motion. All motions are repeated for fifty cycles, with the first three not included in averaging.

Results from a mechanical strike-test by tapping the plate with a metal rod and recording the frequency is shown in Figure 3. The decaying ringing force signal is from the lowest eigenfrequency on the plate setup in water. The frequency content reveals that the eigenfrequency is $15.5 \mathrm{~Hz}$, which is well above the highest forced oscillation frequency of $9 \mathrm{~Hz}$. However, below the eigenfrequency, the amplitude is still slightly frequency dependent. With the knowledge that the response is proportional to frequency squared in this region, ${ }^{13}$ the polynomial

$$
1+0.01 f^{2}
$$

fits the amplitude-frequency response in Figure 3 and is used to correct the force amplitude data.

In Figure 4 the linearity in dynamic response of the loadcell is tested by oscillating a $0.468 \mathrm{~kg}$ steel disc attached to the loadcell in air to simulate the apparent mass of a plate in water. Force signal amplitude is normalized by the mass times acceleration as to produce a linear relationship with frequency squared.

An additional effect we have to consider is the entire span of the plate does not exhibit uniform motion, but instead has slight aeroelastic flutter. High-speed photogrammetry at 500fps with a PCO DiMax camera of the plate tip at a forced oscillation motion of 0.02c amplitude, $9 \mathrm{~Hz}$ motion in Figure 5 reveal a $<110 \%$ tip deflection, where $100 \%$ is the forced 


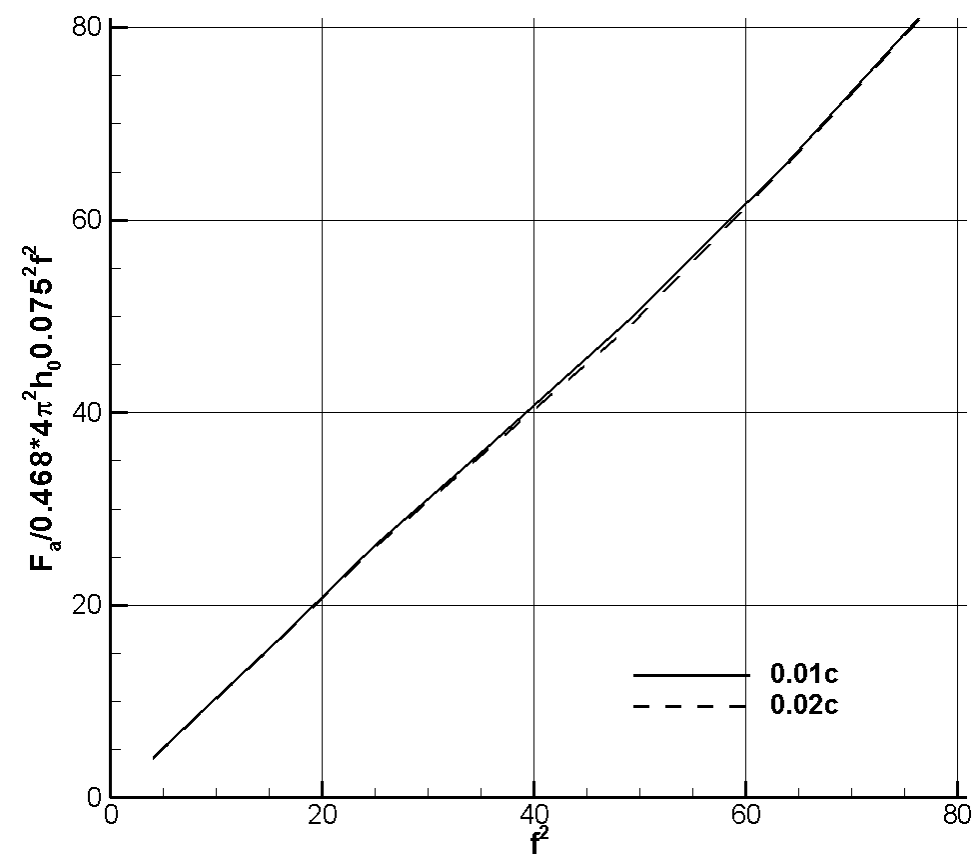

Figure 4: Sinusoidal oscillation of a $0.468 \mathrm{~kg}$ mass in air at two different amplitudes. Force signal amplitude is normalized by the expected force amplitude.
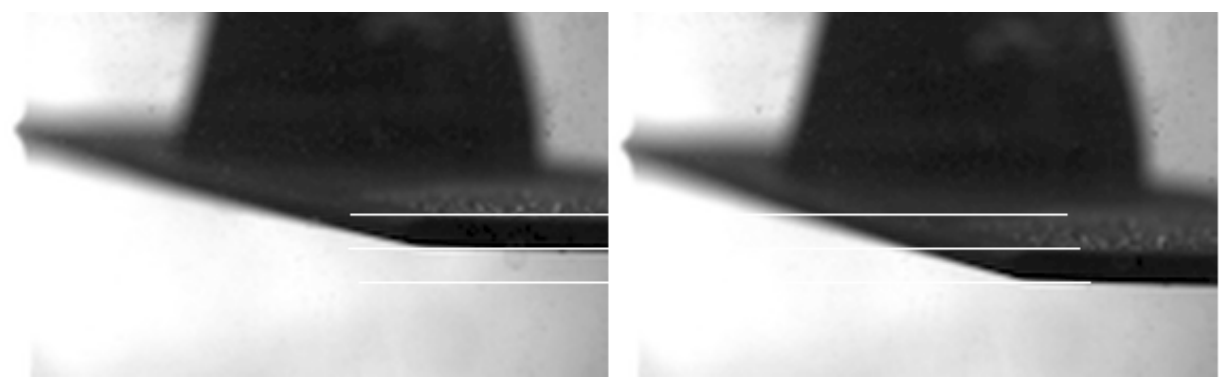

Figure 5: High-speed photogrammetry at $500 \mathrm{~Hz}$ of the plate tip during a $0.02 \mathrm{c}$ amplitude, $9 \mathrm{~Hz}$ forced oscillation motion. Top- (left) and bottom of the motion (right).

amplitude. To investigate this small aerolastic phenomena on the error in measurement of apparent mass, we treat the plate as a cantilevered beam, clamped at the centerline, with a uniformly distributed load in Figure 6.

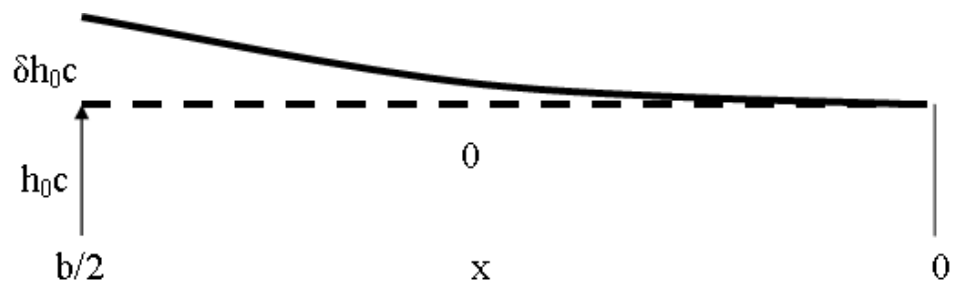

Figure 6: Schematic drawing of a cantilevered plate, clamped at mid-span, oscillated vertically at $h_{0} c$ amplitude with the tip deflecting an extra $\delta h_{0} c$. 
The deflection $\delta h_{0} c$ at a spanwise location $x$ is given by

$$
\delta h_{0} c=\frac{w}{24 E I}\left(x^{4}-4(b / 2)^{3} x+3(b / 2)^{4}\right)
$$

and the maximum defection at the tip $(x=(b / 2))$ from photogrammetry.

$$
\left.\delta h_{0} c\right|_{\max }=\frac{w(b / 2)^{4}}{8 E I}=0.1 h_{0} c
$$

Since apparent mass is proportional to plate amplitude, we use the average aerolastic deflection of the plate as the additional apparent mass.

$$
\delta h_{0, a v g}=\frac{1}{b / 2} \frac{w}{24 E I} \int_{0}^{b / 2)} x^{4}-4(b / 2)^{3} x+3(b / 2)^{4} d x=\frac{1}{3} \frac{w(b / 2)^{4}}{8 E I} \frac{4}{5}=\frac{4}{15} 0.1 h_{0} c=0.0266 h_{0} c
$$

\section{Results}

The theoretical force amplitude on a plate with span $b$ and chord $c$, oscillated sinusoidally at an amplitude $h$ with frequency $f$ is

$$
F_{a}=-\rho \pi b \frac{c^{2}}{4} \frac{\partial u}{\partial t}=\rho \pi b c^{2} h_{0} c \pi^{2} f^{2} \sin (2 \pi f t)=\rho \pi^{3} b c^{3} h_{0} f^{2} \sin (2 \pi f t)
$$

The amplitude of the force signal is normalized by the apparent mass of two single plates such as the force signal is proportional to the oscillation frequency squared $f^{2}$.

$$
\hat{F}=\hat{F}_{m} / \pi^{3} b c^{3} h_{0}
$$

Figure 7 shows the registered force amplitude of a sinusoidal signal from varying the chord-wise spacing of two plates, the oscillation amplitude and frequency against twice the theoretical value of added mass for one plate. One can observe that for plate spacing $=0.5 c$ (bottom right in Figure 7, the registered force amplitude is independent of spacing and oscillation amplitude and follows the linear theoretical prediction well. When decreasing the plate spacing to $d=0.25 \mathrm{c}$ (mid right), the curves show a non-linear behavior with an increasing force amplitude for higher frequencies. There is no dependency of normalized force on oscillation amplitude until the plate spacing is decreased to $d=0.1 c$ (top right). Here the non-linear behavior with frequency is exacerbated further and the oscillation amplitude of $h_{0}=0.05 c$ deviates from the other curves. It is noted that the oscillation amplitude of $h_{0}=0.05 c$ displaces the edge of the plate the same as the $d=0.1 c$ edge-to-edge gap. 

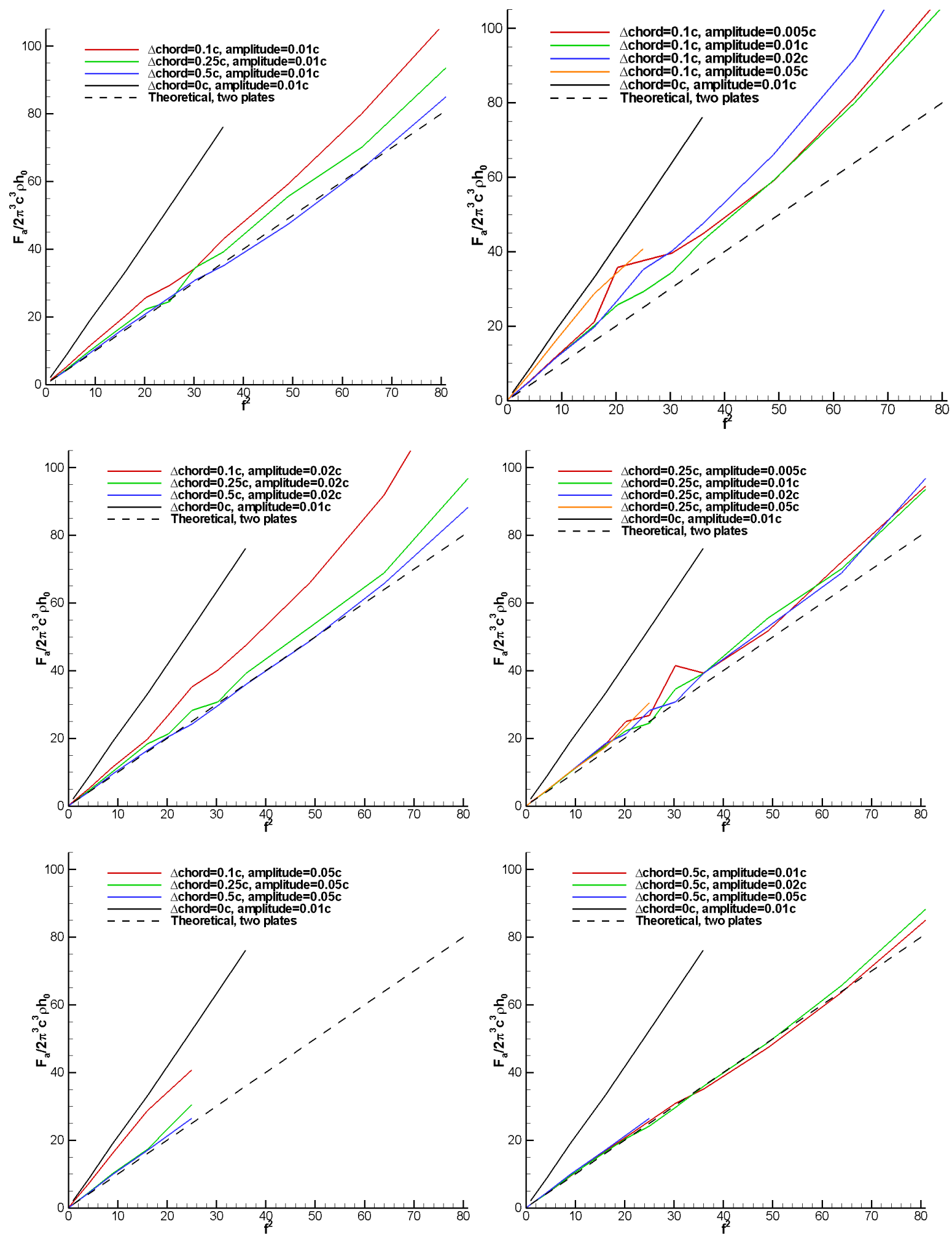

Figure 7: Normalized sinusoidal force amplitude as function of frequency ${ }^{2}$ with varying oscillation amplitude (left column) and varying plate spacing (right column). Dashed black curve is theoretical slope $=1$ for two individual plates. 
When observing the effect of different plate gaps $d$ for the same oscillation amplitude $h_{0}$ in the left column of Figure 7, we can observe a monotonic increasing deviation in slope from the theoretical slope of 1 when the plate gap is reduced. For larger amplitudes $\left(h_{0}=0.05 c\right.$ and $0.025 \mathrm{c}$ in bottom and mid subfigure respectively), the normalized force amplitude slope is closer to the theoretical and for the smaller amplitude of $h_{0}=0.01 c$ (top left). However, the slope deviates faster as the gap is reduced to $d=0.1 c$. At a $d=0.1 c$ gap oscillated at $h_{0}=0.05 c$ amplitude, the force amplitude for the two plates is close to the slope of the no-gap which is is twice that of the theoretical value for two individual plates.
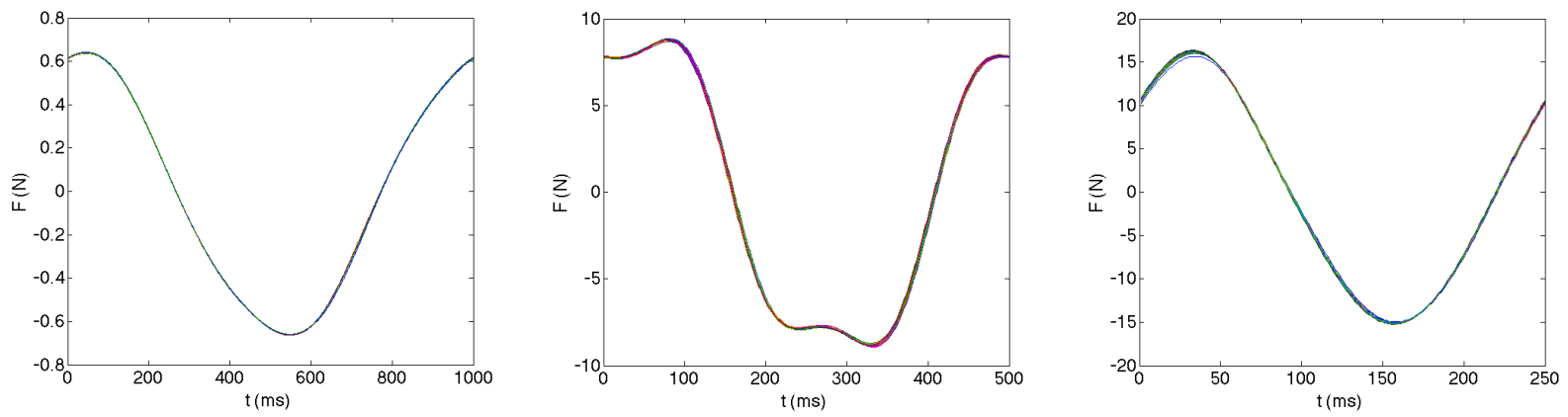

Figure 8: Ensemble of force traces for 48 cycles of the same motion; $2 \mathrm{~Hz}$ (left), $4 \mathrm{~Hz}$ (mid) and $8 \mathrm{~Hz}$ (right), all at $0.02 \mathrm{c}$ amplitude.

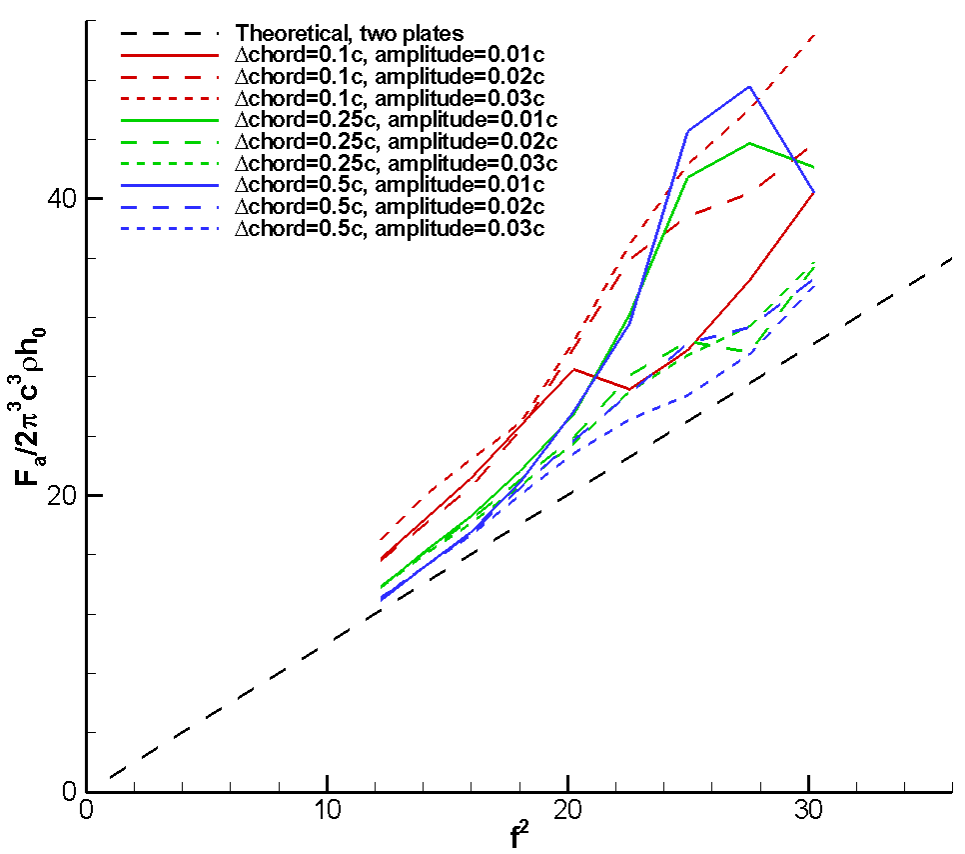

Figure 9: Normalized sinusoidal force amplitude as function of frequency ${ }^{2}$, oscillated at three different amplitudes and frequency $12.25<f^{2}<30.25 \mathrm{~Hz}^{2}$. 
At $\approx 25 H z^{2}(\approx 5 H z)$, the registered force signal is sinusoidal, but with a higher harmonic component as evident in Figure 8. For all tested cases, this harmonic is not a fixed frequency, but scales with the motion frequency indicating a fluid-driven, rather than a structural harmonic. Figure 9 show the amplitude non-linearity for the region where force signal harmonics are present. We observe that larger plate gap together with larger amplitude reduces the effect of the harmonic signal. For higher frequencies $>36 \mathrm{~Hz}^{2}(>6 \mathrm{~Hz})$ and smaller plate spacing than $0.5 \mathrm{c}$, the equivalent added mass is noticeably non-constant while the force signal returns to sinusoidal without harmonics.

A theoretical solution to two cylinders dilating and/or translating has been provided by Wang. ${ }^{12}$ The apparent mass, per cylinder of diameter $c$, for two cylinders at a distance $d$ apart, oscillating in parallel, behaves according to Equation 9 and Figure 10 for large $d$. One can observe that in the limit of $d \rightarrow \infty$, the apparent mass approaches that of a single cylinder 1 and at the $d \rightarrow 0$ limit it is $\frac{\pi^{2}}{3}-1=2.29$ which is also according to Brennen, ${ }^{14}$ unlike 2 as expected for a twice the diameter plate/cylinder. The difference between the analytical and experimental curves are the slope at which apparent mass for two individual objects become gap independent.

$$
F_{p}=-\rho \pi \frac{c^{2}}{4} \frac{\partial u}{\partial t} 4\left(d^{2}-1\right) \sum_{n=1}^{\infty} n \frac{1+e^{-2 n \alpha}}{1-e^{-2 n \alpha}} e^{-2 n \alpha}
$$

where

$$
\alpha=\operatorname{asinh} \sqrt{d^{2}-1}
$$

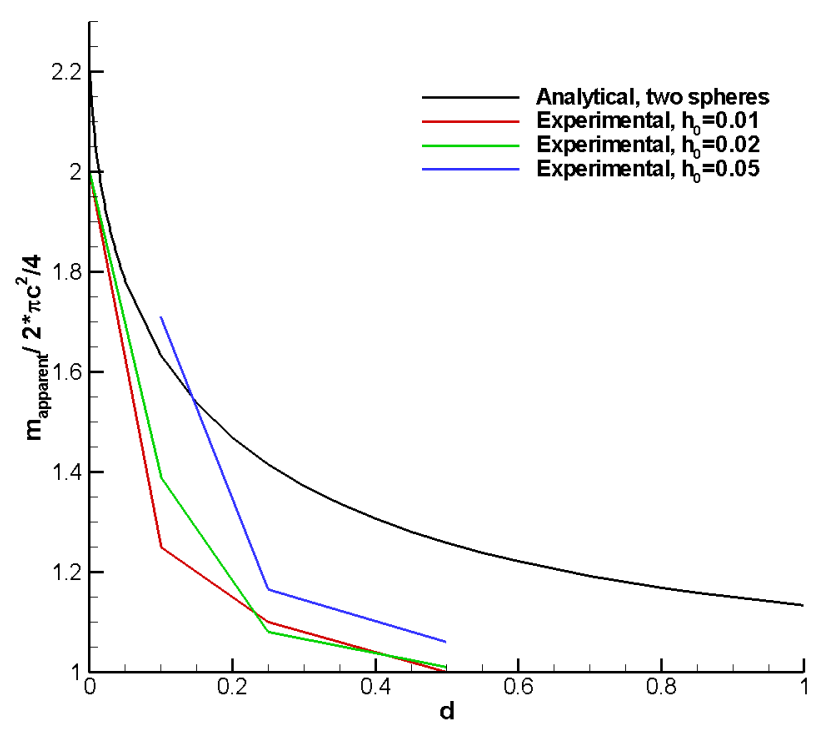

Figure 10: Apparent mass per cylinder when two cylinders are oscillating in parallel with a distance $d$ apart, normalized with the value of a single isolated cylinder. 
The Keulegan-Carpenter ${ }^{16}$ number which describes the importance of viscous effects is given by

$$
K_{c}=\frac{U T}{c}=\frac{h_{0} c 2 \pi f 1 / f}{c}=2 \pi h_{0}
$$

If $K_{C}<<1$, as it is for all cases tested in this paper, it is suggested that viscous effects are not important. However, this conclusion is from a cylinder where the shedding corner is optimally smooth. A flat plate has a much sharper corner for vortices to shed from and a revision to the Keulegan-Carpenter prediction for different cross-section shapes may be warranted.

\section{A. Single plate in freestream}

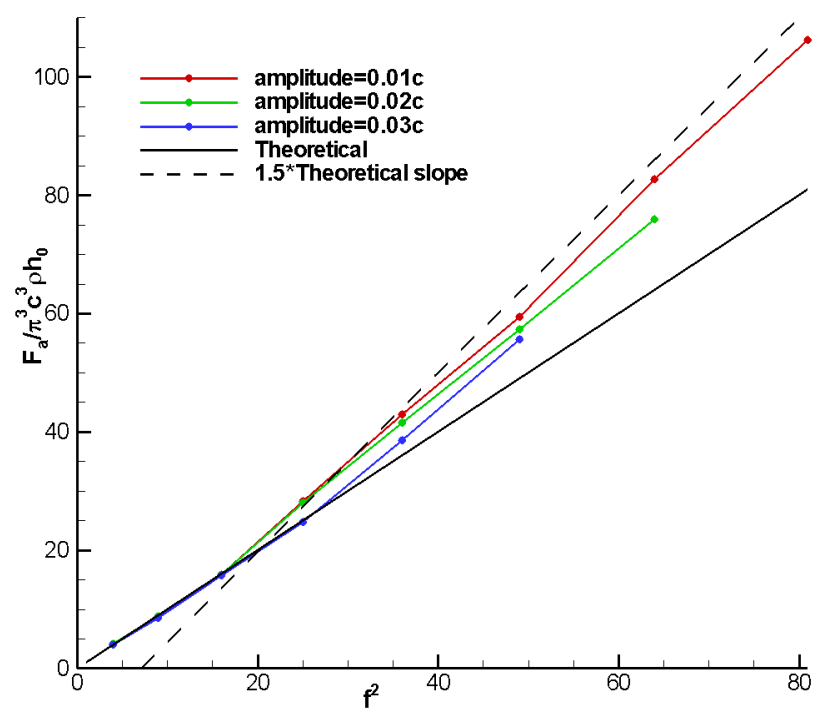

Figure 11: Normal force amplitude for a single plate at $45^{\circ}$ angle of attack in $R e=10 k$ freestream, oscillated at three different amplitudes and frequency $4<f^{2}<81 H z^{2}$.

A single plate oriented at $45^{\circ}$ angle of attack in a $R e=10 k$ freestream is oscillated in normal direction. Because of limitations of physical acceleration for the horizontal linear motor, as the oscillation amplitude is increased, the maximum frequency is reduced.

The measured force has the mean drag subtracted and exhibits the same non-linear response in apparent mass, shown in Figure 11 as the twin plates in Figure 7 . The curves for all three amplitudes break off from the theoretical linear region at $f^{2} \approx 20$ and then asymptotically approach the slope of 1.5 times the apparent mass. The main difference between the single plate in freestram and the twin plates is that the single plate exhibits bluff-body shedding in the wake. Fage and Johanssen ${ }^{15}$ investigated the shedding frequency of the wake for flat plates at angles of attack in freestream. With Equation 12, the shedding frequency is $0.372 \mathrm{~Hz}$, or $0.138 \mathrm{~Hz}^{2}$, which is more than an order of magnitude lower than 
where the experimentally obtained force curves start deviating from the theoretical slope. Thus, it is not believed that the non-linearity of the apparent mass is directly related to the bluff-body wake, even though the off-body vortex dynamics may still play a role in fluid acceleration.

$$
S t_{F J}=\frac{f \operatorname{csin}(\alpha)}{U_{\infty}}=0.148
$$

\section{References}

${ }^{1}$ Theodorsen, T., "General theory of aerodynamic instability and the mechanism of flutter," NACA $R-496,1935$.

${ }^{2}$ Küssner, H., "Untersuchung der Bewegung einer Platte beim Eintritt in eine Strahlgrense," Luftfahrtforschung, Vol. 13, No. 425, 1936.

${ }^{3}$ von Karman, T. and Sears, W. R., "Airfoil Theory for Non-Uniform Motion," Journal of the Aeronautical Sciences, Vol. 5, No. 10, August 1938, pp. 379-390.

${ }^{4}$ Garrick, I. E., "On some reciprocal relations in the theory of nonstationary flows," NACA Report, , No. 629, 1938.

${ }^{5}$ Greenberg, J. M., "Airfoil in Sinusoidal Motion in a Pulsating Stream," Tech. Rep. 1326, NACA Technical Report, June 1947.

${ }^{6}$ Lighthill, M., "On the Weis-Fogh mechanism of lift generation," Journal of Fluid Mechanics, Vol. 60, 1973, pp. 1-17.

${ }^{7}$ Chow, C.-Y. and Huang, M.-K., "The initial lift and drag of an impulsively started airfoil of finite thickness," Journal of Fluid Mechanics, Vol. 118, 1982, pp. 393-409.

${ }^{8}$ Graham, J. M. R., "The lift on an aerofoil in starting flow," Journal of Fluid Mechanics, Vol. 133, 1983, pp. 413-425.

${ }^{9}$ Pullin, D. I. and Wang, Z. J., "Unsteady forces on an accelerating plate and application to hovering insect flight," Journal of Fluid Mechanics, Vol. 509, No. -1, 2004, pp. 1-21.

${ }^{10}$ Ramesh, K. K., Gopalarathnam, A., Ol, M. V., Granlund, K. O., and Edwards, J., "Augmentation of Inviscid Airfoil Theory to Predict and Model 2D Unsteady Vortex Dominated Flows," AIAA Paper 2011$3578,2011$.

${ }^{11}$ Granlund, K. O., Ol, M. V., and Bernal, L., "Unsteady pitching flat plates," Journal of Fluid Mechanics, Vol. 733, October 2013.

${ }^{12}$ Wang, Q. X., "Interaction of two circular cylinders in inviscid fluid," Physics of Fluids, Vol. 16, No. 12, December 2004, pp. 4412-4425.

${ }^{13}$ Doebelin, E. O., Measurement Systems: Application and Design, McGraw-Hill, 4th ed., 1990.

${ }^{14}$ Brennen, C. E., "A Review of Added Mass and Fluid Inertial Forces," Tech. Rep. CR 82.010, Naval Civil Engineering Laboratory, Port Hueneme, CA 93043, January 1982.

${ }^{15}$ Fage, A. and Johansen, F. C., "On the Flow of Air behind an Inclined Flat Plate of Infinite Span," Proceedings of the Royal Society, Vol. 116, No. 773, 1927, pp. 170-197.

${ }^{16}$ Keulegan, G. H. and Carpenter, L. H., "Forces on Cylinders and Plates in an Oscillating Fluid," National Bureau of Standards, Vol. 60, No. 5, 1956, pp. 423-440. 